\title{
2D Materials Characterization: Should We Rely on HR STEM Imaging?
}

Sergei Lopatin ${ }^{1}$, Vladimir Roddatis ${ }^{2}$, Tobias Meyer ${ }^{3}$ Areej Aljarb ${ }^{4}$, Lain-Jong $\mathrm{Li}^{4}$ and Vincent Tung ${ }^{4}$

1. King Abdullah University of Science and Technology (KAUST), Core Labs, Thuwal, Saudi Arabia.

2. Institute of Materials Physics, University of Goettingen, Goettingen, Germany.

3. 4th Institute of Physics - Solids and Nanostructures, University of Goettingen, Goettingen, Germany.

4. Physical Sciences and Engineering Division, King Abdullah University of Science and Technology (KAUST), Thuwal, Saudi Arabia.

The strictly two-dimensional materials, such as graphene, transition metal dichalcogenides (e.g., $\mathrm{MoS}_{2}$, $\mathrm{WSe}_{2}$ ), metal oxides, and the emerging Mxenes, exhibit exceptionally high crystal and electronic quality, and, despite its short history, has already revealed a cornucopia of new physics and potential applications. High resolution (S)TEM imaging techniques for materials characterization gaining unprecedented spatial resolution with the development of spherical aberration (Cs) correctors naturally migrated from "classical" bulk samples to their 2D counterparts. However, it is the microscopy of these "super thin" materials, which very often consist of a few or even single atom monolayers, may introduce some imaging artifacts with a dramatic effect on the structure determination.

In this study, we characterize the quality of $\mathrm{MoS}_{2}\left(\right.$ and $\left.\mathrm{WS}_{2}\right)$ monolayer films grown by chemical vapor deposition (CVD). It was established that 2D grains grow as triangular shape patches with only 2 possible orientations having approximate $180^{\circ}$ in-plane rotation mismatch (antiphase). Other than the "opposite" orientation these two types of grains show no evidence of different physical properties, which was confirmed by XPS, photo-luminescence and EELS bandgap measurements. When grains grow bigger, eventually they merge into continuous monolayer film forming significant number of antiphase $\left(180^{\circ}\right)$ grain boundaries. The quality and structure of the grain boundary interfaces were further characterized by HR STEM imaging performed at $80 \mathrm{kV}$ with a ThermoFisher USA (former FEI Co) Titan Themis Z (40-300kV) TEM equipped with a double Cs corrector, a high-brightness electron gun (x-FEG), and an electron beam monochromator.

Despite the fact that the material on both sides from the interface is the same, its contrast in HR STEM images appears significantly different (Fig.1). Namely, the left-hand side demonstrates features typical for the so-called $2 \mathrm{H}$ phase monolayer $\mathrm{MoS}_{2}: 3 \mathrm{Mo}$ atoms and 3 pairs of $\mathrm{S}$ atoms form 6-fold rings. At the same time, the contrast of $\mathrm{S}$ atoms on the right-hand side is significantly reduced, leaving only Mo atoms visible, which appears as the so-called 1T phase. This effect of Lighter Elements Contrast Degradation (LECD) correlates to the orientation (rotation) of the sample inside the TEM holder. Thus, by in-plane (orthogonal to the electron beam) rotation of TEM grid inside the TEM holder it is possible to find the orientation for which the LECD effect is absent - zero LECD orientation - in which case the material on both sides from the interface have similar $2 \mathrm{H}$ type contrast only. Rotation to $+/-30^{\circ}$ from zero results in the maximum LECD effect. However, the contrast of $+30^{\circ}$ and $-30^{\circ}$ rotations is "reversed". In other words, if a certain area of the sample (for example right-hand side in Fig.1) appears as $1 \mathrm{~T}$ phase for $-30^{\circ}$ rotation, exactly the same area appears as $2 \mathrm{H}$ phase for $+30^{\circ}$ rotation (and vice versa for the left-hand side). Further rotation to high angles proves 3 -fold $\left(120^{\circ}\right)$ in-plane rotation symmetry of the LECD effect. Moreover, the LECD effect is dependent on the imaging system chromatic aberration. For the monochromated electron beam (in our experiments the energy spread was below $70 \mathrm{meV}$ ) the LECD effect can be reduced below the detection limit. 
At the same time the LECD effect does not depend on STEM defocus, slight (few degrees) TEM grid mistilt or position on the grid itself. For a given sample orientation (for example $-30^{\circ}$ ) all the interfaces across the TEM grid with the same orientation of the grains growth (antiphase) mismatch show identical $2 \mathrm{H}-1 \mathrm{~T}$ like contrast and there are no defocus values to image it in "reversed", i.e. 1T-2H like way.

For the root cause analysis of the LECD effect we performed HR STEM imaging simulations by employing QSTEM [1] and Dr. Probe [2] software packages. We addressed the effect on light elements STEM contrast formation taking into account: a) non-uniform radial gain of the Annular Dark Field detector (Fischione) and b) presence of the residual low order aberrations in the STEM probe forming system. We demonstrate that the LECD effect may be almost perfectly matched (including $120^{\circ}$ rotation symmetry) with simulations if just a small amount (about 100nm) of 3-fold astigmatism is introduced (not corrected) to the Cs corrected STEM probe. The simulations are applied also to predict the imaging artefacts for other 2D materials. On the basis of the combined imaging characterization, we have arrived at the conclusion that indicates the need for employing more critical approach for interpretation of HR STEM imaging results, especially while dealing with 2D materials. The finding also calls scientists and engineers to carefully visualize, comprehensively characterize, and then conclusively rationalize the rapidly expanding forefronts of emerging 2D materials on a firm scientific footing.

\section{References:}

[1] CT Koch, Determination of Core Structure Periodicity and Point Defect along Dislocations, Arizona State University, Tempe, USA; (2002).

[2] J Barthel, Ultramicroscopy 193 (2018), p.1.

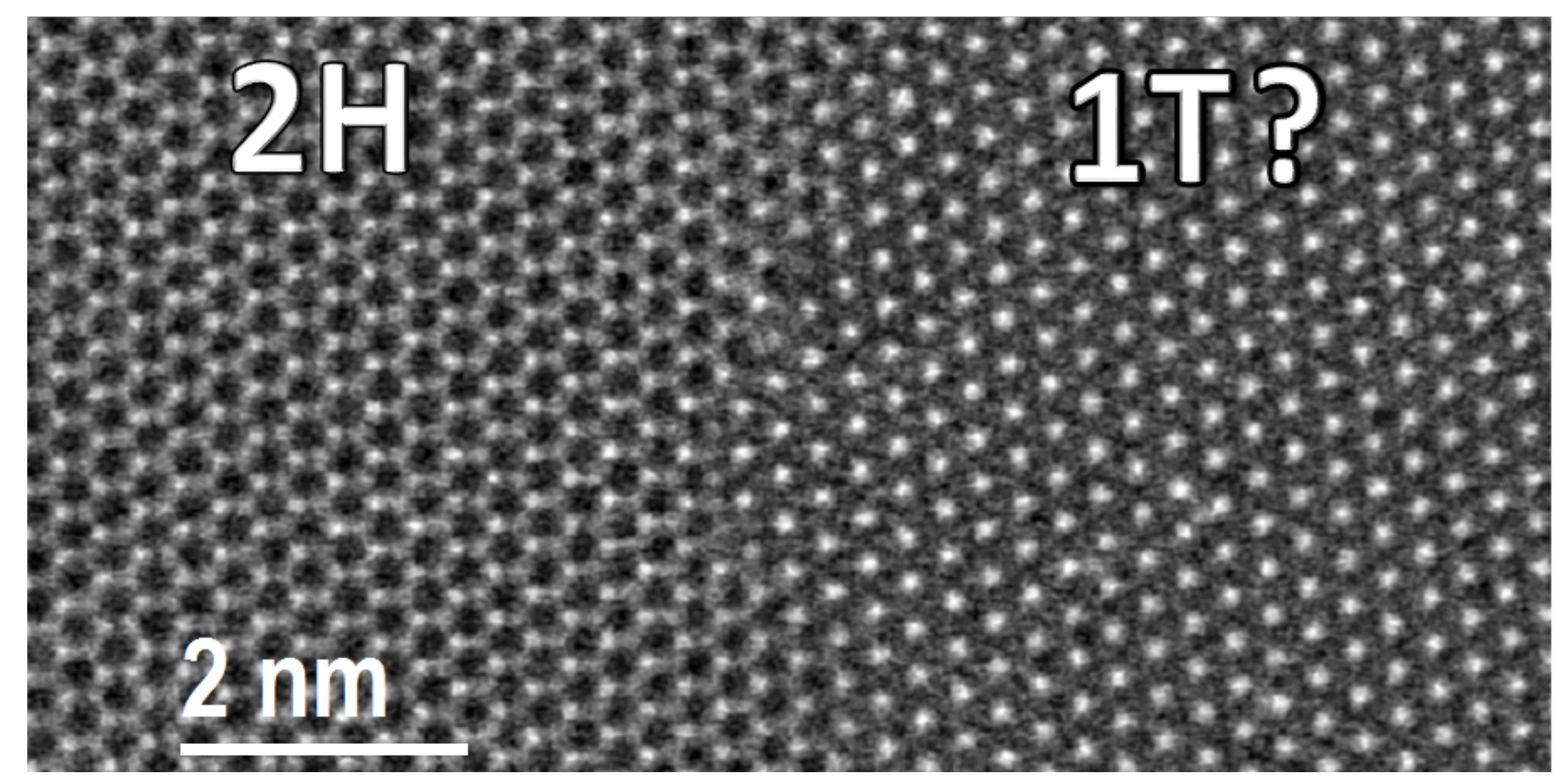

Figure 1. HR STEM image of the interface between a monolayer $\mathrm{MoS}_{2}$ grains which appear as different $2 \mathrm{H}$ (left) and $1 \mathrm{~T}$ (right) phases. However, both grains are proven identical (2H phase) with about $177^{\circ}$ in-plane rotation mismatch. 